Presented at the Fall, 1994 MRS meeting, Nov. 28, 1994, Boston

Submitted for MRS proceedings, vol. 359

\title{
Field Emission and Growth of Fullerene Nanotubes
}

Andrew G. Rinzler, Jason H. Hafner, Pavel Nilolaev, Daniel T. Colbert and Richard E. Smalley Rice Quantum Institute and Departments of Chemistry and Physics, Rice University, Houston, Texas 77251.

\section{ABSTRACT}

Efforts to control the growth of in đividual cărbon nanotubes from nanotube seed crystals have led to a characterization of their field-induced electron emission behavior. The application of a bias voltage in our growth apparatus was motivated by the prolific formation of nanotubes in the carbon arc growth method, in which the electric field appears to play a central role. ${ }^{1,2} \mathrm{We}$ report here the ability to achieve various tube tip configurations by the controlled application of voltage, heat and chemicals to an individual nanotube, and that these states are well characterized by the emission currents they induce.

\section{BACKGROUND}

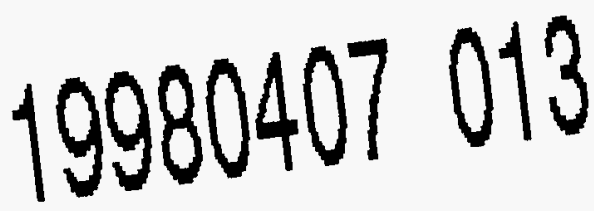

Much progress has been made in increasing their yield, ${ }^{3}$ and in purification, ${ }^{4,5}$ but substantial quantities of carbon nanotubes are still not generally available with a perfect graphene structure over sufficient lengths that they truly deserve the term fullerene fibers. ${ }^{6}$ For example, nanotubes are produced in great quantity by the carbon arc method, but appear always to contain defects over lengths beyond several microns. Attempts to ameliorate this have recently led us to propose a model for growth in the carbon arc, and to the conclusion that defects unavoidably arise from tubes' sintering together in the extreme heat of the arc. ${ }^{2}$ To circumvent this problem and to discern growth conditions which will yield defect-free nanotubes of any desired length we have-engaged in experiments designed to perpetuate the growth of a single nanotube, starting from a nanotube seed crystal collected from the carbon arc deposit.

In order to place our field emission results in context, it is useful to revisit some of our other more recent findings in this fast breaking field. A key feature of our carbon arc growth model is the synergetic relationship between the growing nanotubes and the arc plasma. Here the $10 \mathrm{~V}$ potential drop across the very thin ( $<1$ micron) "cathode layer" generates a high electric field which, when further concentrated in the vicinity of the exceptionally sharp nanotube tips may be as high as $1 \mathrm{~V} / \AA$. Under such conditions the (growing) open tips of the nanotubes act as highly effective field emitters -- so effective that this field emission becomes the dominant mechanism for electron injection into the arc plasma. In response, the carbon ion density and current flow in the plasma become concentrated in the region above the emitting tubes. The high

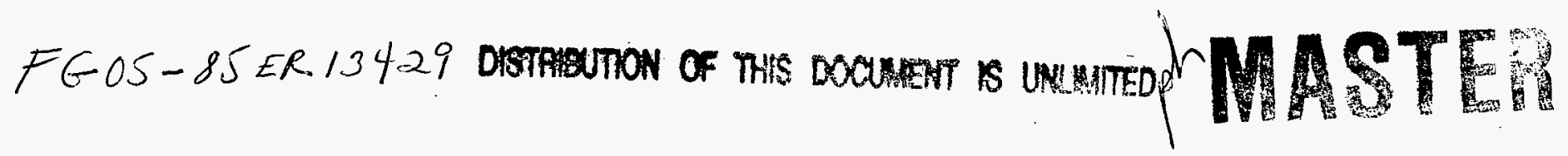


electric field in the vicinity of the tips further serves then to attract the positive carbon ions, : $\xi$ which in this region provide the dominant feedstock for nanotube growth.

We have previously maintained that the high electric field is also critical in preventing the growing end of the nanotube from closing off with a fullerene cap and thus terminating further growth. Calculations have indicated, ${ }^{7}$ however, that a field sufficient to keep a single walled nanotube open would be more than sufficient to break $\mathrm{C}-\mathrm{C}$ bonds at the open tube end, leading to a wholesale field-evaporation of carbon from the tip. The question then remains what mechanism keeps the tubes from closing. Recently reported experiments from our group, ${ }^{8}$ in which nanotubes were discovered under conditions previously thought to exclude their growth, have shed new light on this question.

In these experiments, nanotubes were found in the same the laser-vaporization apparatus that produces high yields of spheroidal fullerenes. In order to rationalize nanotube growth at a temperature high enough $(1200 \mathrm{C})$ to anneal closed spheroidal fullerenes, we were forced to invoke an intrinsic factor which inhibits the closure of a forming nanotube. We proposed that for a multilayer nucleating "prototube" the highly reactive and energetic dangling bonds at the growing open edges interact with one another, most likely with bridging atoms between adjacent layers. The incentive to eliminate dangling bonds by closing diminishes once they become partially deactivated in this way. For a multiwall nanotube, the open structure can be energetically lowered enough that the energetic barrier to closure allows lengthening if feedstock adds on a time scale rapid compared with the rate of closure. (By "open", we now mean the general case where the inner hole is open, rather than that each edge atom exposes dangling bonds.) In this regard, it is significant that we observed no single-layer nanotubes in the laservaporization apparatus, since the model predicts that any single-layer shell lacks the stabilizing interactions required to lengthen, and will readily anneal closed. The energy-lowering by the electric-field (roughly $2-3 \mathrm{eV}$ for the fields in the arc), combined with the stabilization conferred by bridging species may now be sufficient to keep the tips open for growth in the arc.

These considerations prompted the application of an electric field to the nanotubes when mounted in our growth apparatus, leading to a study of field-emission as a diagnostic for the state of the nanotube growth site, its tip. Questions we may now address are: What is the precise nature of an "open" tip? How does an electric field affect the relative stabilities of open and closed tips? Can we know the state of the tube tip without subjecting it to a destructive test, allowing growth experiments to be performed under controlled conditions?

\section{FIELD EMISSION OF NANOTUBES}

We shall argue below that field-emission can be used to infer the configuration of a nanotube tip. Ideally, we would like to confirm our model tying the state of the nanotube tip to its emission behavior by directly imaging the tip in the TEM. Unfortunately, experimental circumstances conspire to make this difficult at present. In our growth apparatus, the length of a nanotube mounted on the supporting electrode must be sufficiently long to heat its tip to incandescence by a focused laser beam without illuminating the much larger electrode. For nanotubes which are too short scattering from the electrode makes it difficult to differentiate, with our imaging equipment, the image of the incandescing nanotube (even with filtering to reject the laser light). When a nanotube of this free-standing length (attached to an electrode) is mounted in a specially designed pallet in our TEM we have found that while the resolution is 


\section{DISCLAIMER}

This report was prepared as an account of work sponsored by an agency of the United States Government. Neither the United States Government nor any agency thereof, nor any of their employees, make any warranty, express or implied, or assumes any legal liability or responsibility for the accuracy, completeness, or usefulness of any information, apparatus, product, or process disclosed, or represents that its use would not infringe privately owned rights. Reference herein to any specific commercial product, process, or service by trade name, trademark, manufacturer, or otherwise does not necessarily constitute or imply its endorsement, recommendation, or favoring by the United States Government or any agency thereof. The views and opinions of authors expressed herein do not necessarily state or reflect those of the United States Government or any agency thereof. 
adequate to observe that we do indeed have a single nanotube at the tip (and can estimate its: diameter), it is not sufficient to permit resolution of the tube wall fringes necessary to judge the state of the tip. Since we routinely image the walls of nanotubes mounted on holey carbon grids, we at first suspected the problem was mechanical vibrations acting on the long, unsupported length of the electrode mounted tubes. Closer consideration, however, has led us to the conclusion that the limitation is more fundamental in that on the nanometer scale, thermal vibrations become significant.

Modeling the nanotube as a continuous beam of length $l$ and Young's modulus $Y$, rigidly fixed at one end, one can calculate its deflection, $\Delta z$, at the free end when subjected to a point force from elasticity theory. ${ }^{9}$ The energy required to displace the beam tip by a small displacement $\Delta \mathrm{z}$ is $\Delta \mathrm{E}=3 \pi \mathrm{Yr}^{4} \Delta \mathrm{z}^{2} / 8 l^{3}$, where $\mathrm{r}$ is the beam radius, and the area moment for a solid, cylindrical beam has been assumed. Setting the energy available for bending to $\mathrm{kT}$ by equipartition (the bending mode is doubly degenerate), and using the Young's modulus of Iridium (a particularly stiff material; nanotubes are thought to be stiffer yet), $Y=5.2 \times 10^{11} \mathrm{~N} / \mathrm{m}^{2}$, a nanotube of radius $10 \mathrm{~nm}$ and length 10 microns is calculated to thermally deflect $26 \mathrm{~nm}$ at its tip at room temperature. This easily accounts for the observed lack of resolution in our electrode mounted nanotube TEM images. We are at present working to develop the techniques for reliably transferring a nanotube sample from an electrode to a TEM grid. Until such time our inferences concerning the various states of nanotube tips must rely on the evidence presented here.

\section{Experimental}

The electrode, to which the nanotubes are attached, consist of a short length ( $-4 \mathrm{~mm}$ ) of a commercially available 8 micron diameter carbon fiber, one end of which has been attached by conductive silver paint to a small steel pallet for handling and mounting in our TEM, SEM or growth apparatus. Attachment of a nanotube to the free end of the carbon fiber proceeds as follows: a small piece of arc grown, purified boule ${ }^{2}$ is mounted on an $X-Y-Z$ translation stage within the field of view of a high power $(800 \mathrm{X})$ optical microscope set up for reflected dark field operation. Since individual nanotubes, and even bundles of adjacent nanotubes, are far smaller in diameter than the wavelengths of visible light it would hardly seem likely that these could be imaged by an optical system, however, for the component of the incident light polarized along the length of the tubes we have found them to be quite efficient scatterers and hence readily visible under these conditions. The boule will typically possess numerous bundles or "stalks" of nanotubes which stick out further than others. With its pallet attached to an opposing X-Y-Z translation stage the commercial carbon fiber is now maneuvered so that it is positioned adjacent to a selected stalk with several microns of overlap. The two are now brought into contact and the commercial fiber is withdrawn. Frequently, the van der Waals bonding of this stalk to the commercial fiber is sufficient to cause the stalk to go with the fiber, thus pulling out of the boule.

Often, the intensity of the light scattered from the stalk, now connected to the fiber, is seen to diminish in stages as one follows along its length to the free end. When the final stage at the tip of the stalk is exceptionally straight and barely visible, indicating it to be very thin, TEM images have confirmed that the stalk has tapered down to a single nanotube. The stalks range in lengths up to 60 microns with the average being 14 microns. In cases where the lengths of the free-traveling single nanotube at the tip are less than 5 microns the sample is rejected. The typical sample we use has a single tube, free-travel length of $\sim 8$ microns although these have 


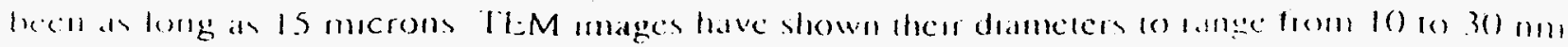

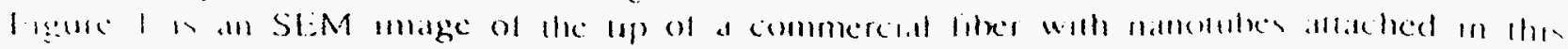
minumit

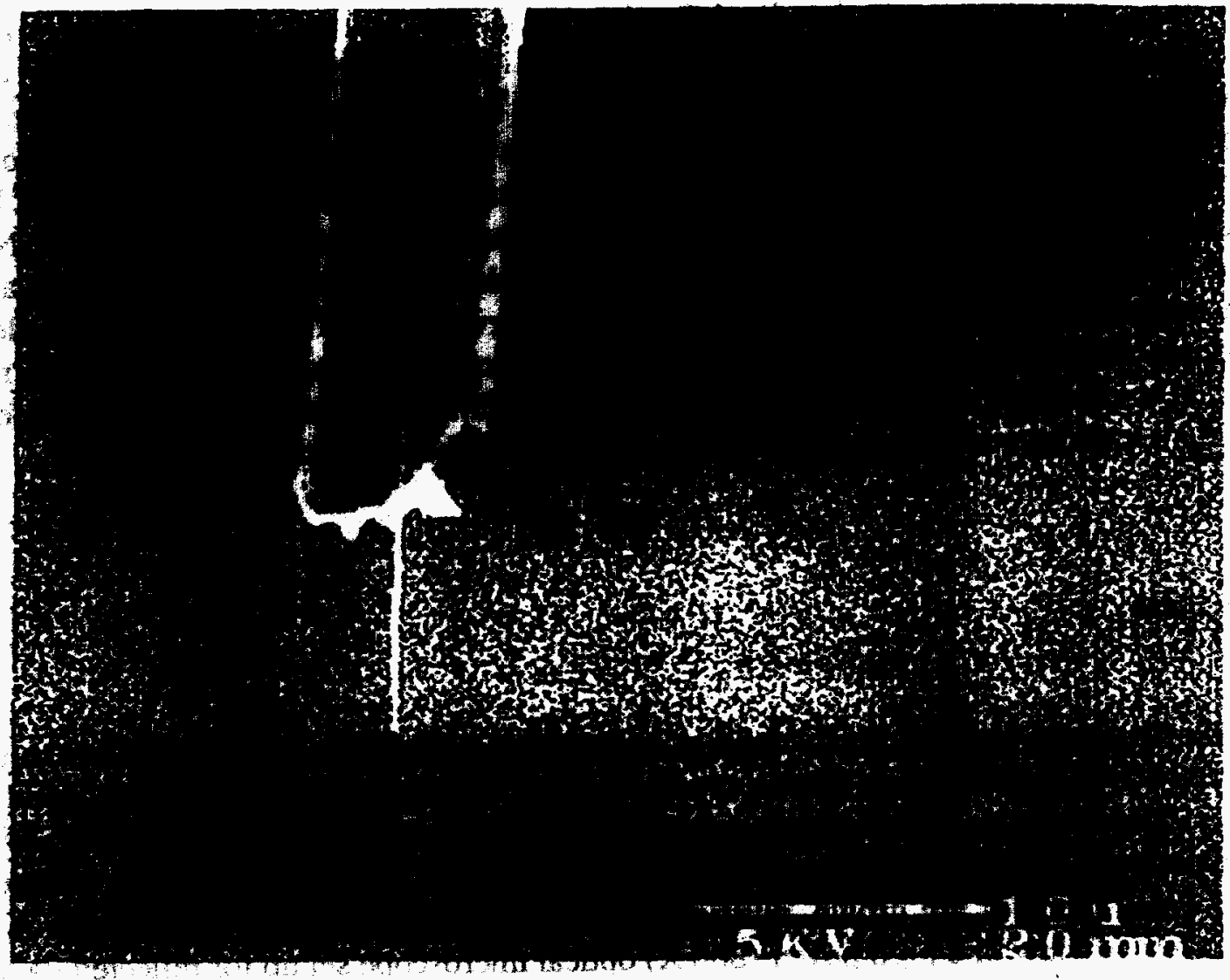

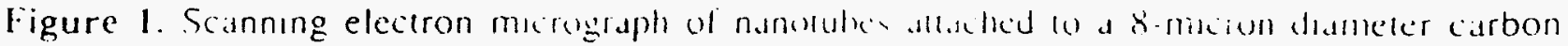

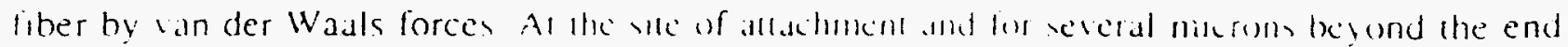

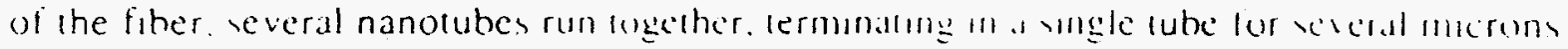

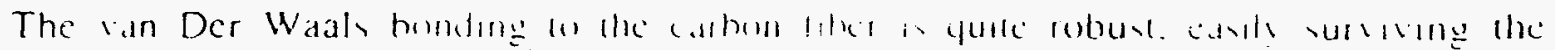

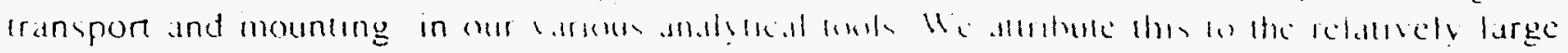

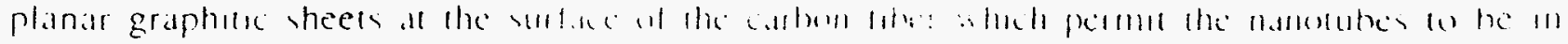

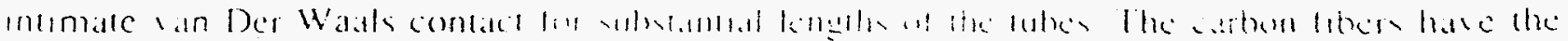

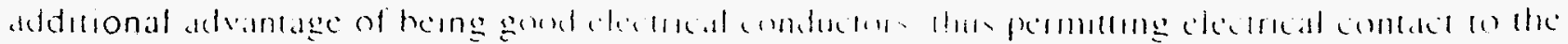
n.tnotuhes

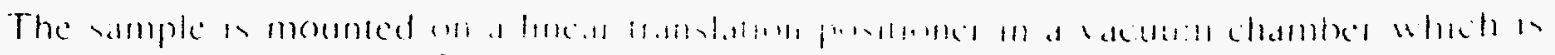

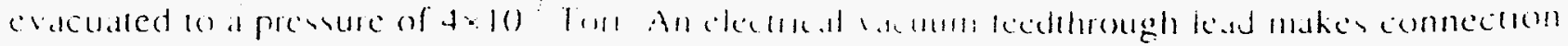

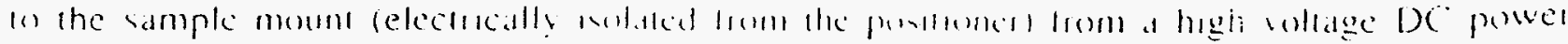

\section{Reproduced From Best Available Copy}


supply. A second electrical feedthrough lead connects to the opposing electrode which consists of : an otherwise electrically isolated brass cup $(-3 \mathrm{~mm}$ diam.) set $\sim 1 \mathrm{~mm}$ from the nanotube. This connects the cup to electrical ground through a Keithley model 345 picoammeter which monitors the emitted electron current.

A $10 \mathrm{~cm}$ focal length lens mounted on a precision $X-Y-Z$ translation stage focuses the $514.5 \mathrm{~nm}$ laser light of a CW Ar-ion laser to a 5 micron Gaussian spot at the tip of the nanotube. Visual monitoring of the tube is accomplished with a Microzoom $I$ microscope fitted with a long working distance $50 \mathrm{X}$ objective (N.A. 0.45) situated at right angles to both the nanotube long axis and the direction of the exciting beam. The scattered green light or incandescent radiated light image of the nanotube is observed using a Princeton Instruments back lit CCD camera sensitive out to 1 micron in the near infrared.

$\underline{\text { Results and Discussion }}$

Figure 2 displays typical data of emission current as a function of applied voltage. Initially, the onset voltage (i.e., the bias voltage yielding the smallest detectable current, which in our apparatus is $0.1 \mathrm{pA}$ ) is high, here about $-120 \mathrm{~V}$ (negative bias). We believe this state corresponds to a nanotube tip overcoated with an amorphous layer; such a blunt tip enhances the local electric field much less than one with sharper features. (In this figure, we have plotted for reference the Fowler-Norheim ${ }^{10}$ field-emission curves for metallic tips of various radii, assuming a work function of $5.0 \mathrm{eV}$.) After laser-heating the nanotube tip to $1000-2000 \mathrm{C}$ for 1-2 minutes to desorb the overcoating, the emission onset falls to about $-80 \mathrm{~V}$ (middle curves), and was found to be reproducible if the current was not taken above several $\mathrm{nA}$. When sufficient bias voltage was applied to induce a current on the order of $1 \mu \mathrm{A}$, the onset dropped to about $-65 \mathrm{~V}$. With zero voltage, the tip was laser-annealed at 1000-2000 C for 1-2 minutes, and the intermediate emission onset of $-80 \mathrm{~V}$ was recovered. We believe that the different emission onsets are characteristic of different states of the emitting tip: the $-80 \mathrm{~V}$ onset corresponds to a closed tip, while the lower onset corresponds to an open tip whose sharper features enhance the local electric field. Included in the figure are emission data recorded the day after leaving the tip in the open state overnight. The onset potential of this state was then found to be $-65 \mathrm{~V}$ as before, indicating its stability under vacuum. In summary, the voltage onset for emission depends on the history of the nanotube.

At emission currents of $\sim 1 \mu \mathrm{A}$ and higher, the nanotube tip is seen to glow. In cases where a higher voltage is required to achieve currents of this scale (indicating a fatter tube tip) the nanotube has been observed to shorten at a rate of about $1 \mu / \mathrm{min}$. and faster. In such cases the emitted light intensity increases substantially. We attribute this emitted light to radiation due to ohmic heating of the tip as electrons scatter off phonons in that region. That this light is confined to the tip we ascribe to differences between the tube walls and the tip as regards their respective electron-phonon interactions. The sudden increase in the emitted radiation observed when the nanotube is seen to shorten we attribute to the opening of a formerly closed tube which shortens due to field-desorption of its carbon walls. The increase in the light intensity derives 


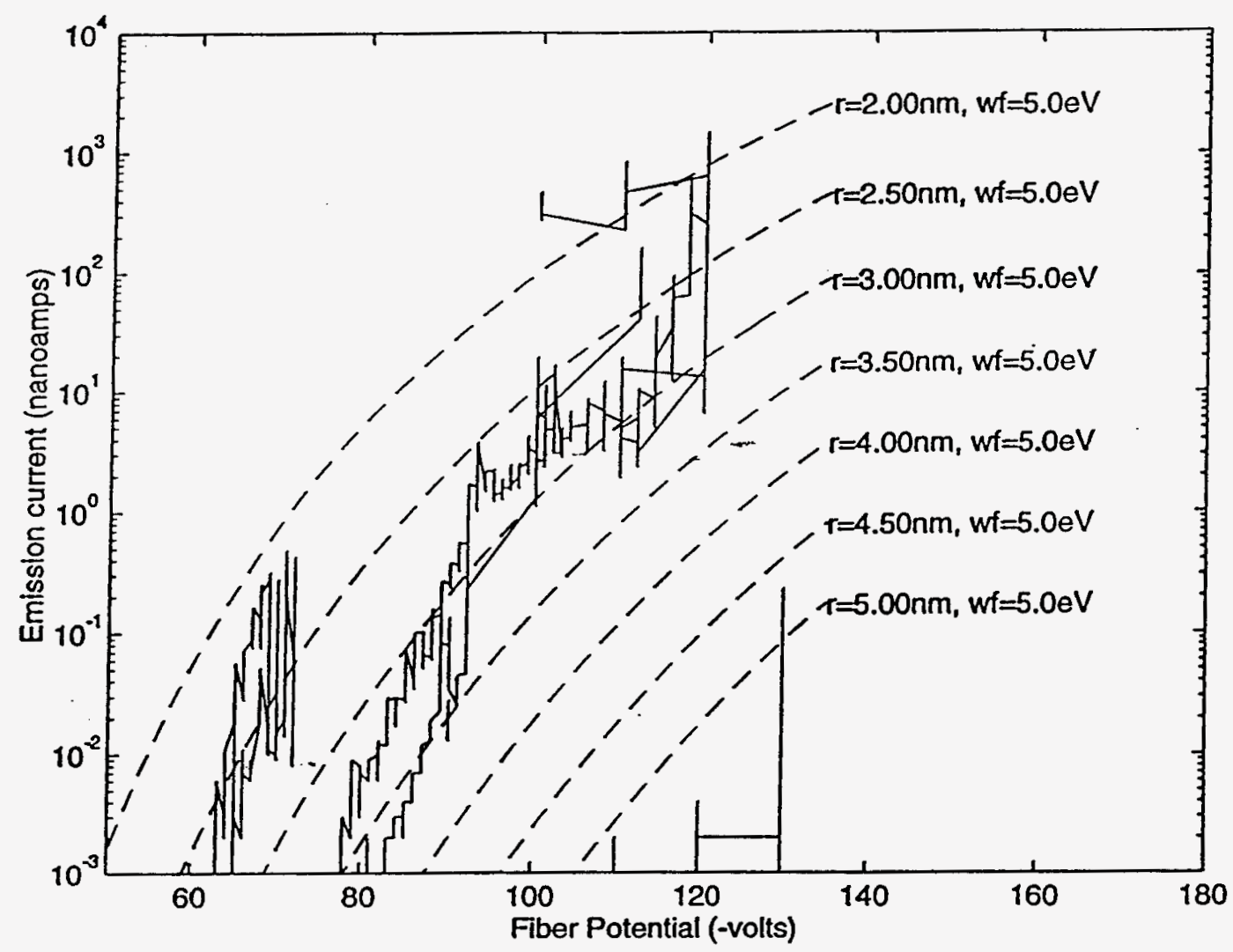

Figure 2. Emission current $v s$. applied bias voltage for a single nanotube.

from heating of the tip by the violent recoil of atoms left behind as edge atoms are torn away by the field.

We can achieve the open tip state non-destructively by exposing the nanotube to $5 \mathrm{mTorr}$ $\mathrm{O}_{2}$ at $500-1000 \mathrm{C}$ for several minutes. Figure 3A shows the emission behavior prior to such oxidative opening. Shown is the field emission current at $-83 \mathrm{~V}$ bias as a function of time; with the laser beam which heats the tube tip to $\approx 1000 \mathrm{C}$ repeatedly blocked and then unblocked. That the emission current is substantially increased when the laser heating is in effect is readily understood as a simple thermal enhancement of this field induced emission. Figure 3B, in contrast, shows the behavior under similar conditions for this nanotube after oxidative opening. Note that there continues to be a difference in the magnitude of the emission with the laser blocked and unblocked, however, the higher emitting state now pertains to when the laser is blocked! 


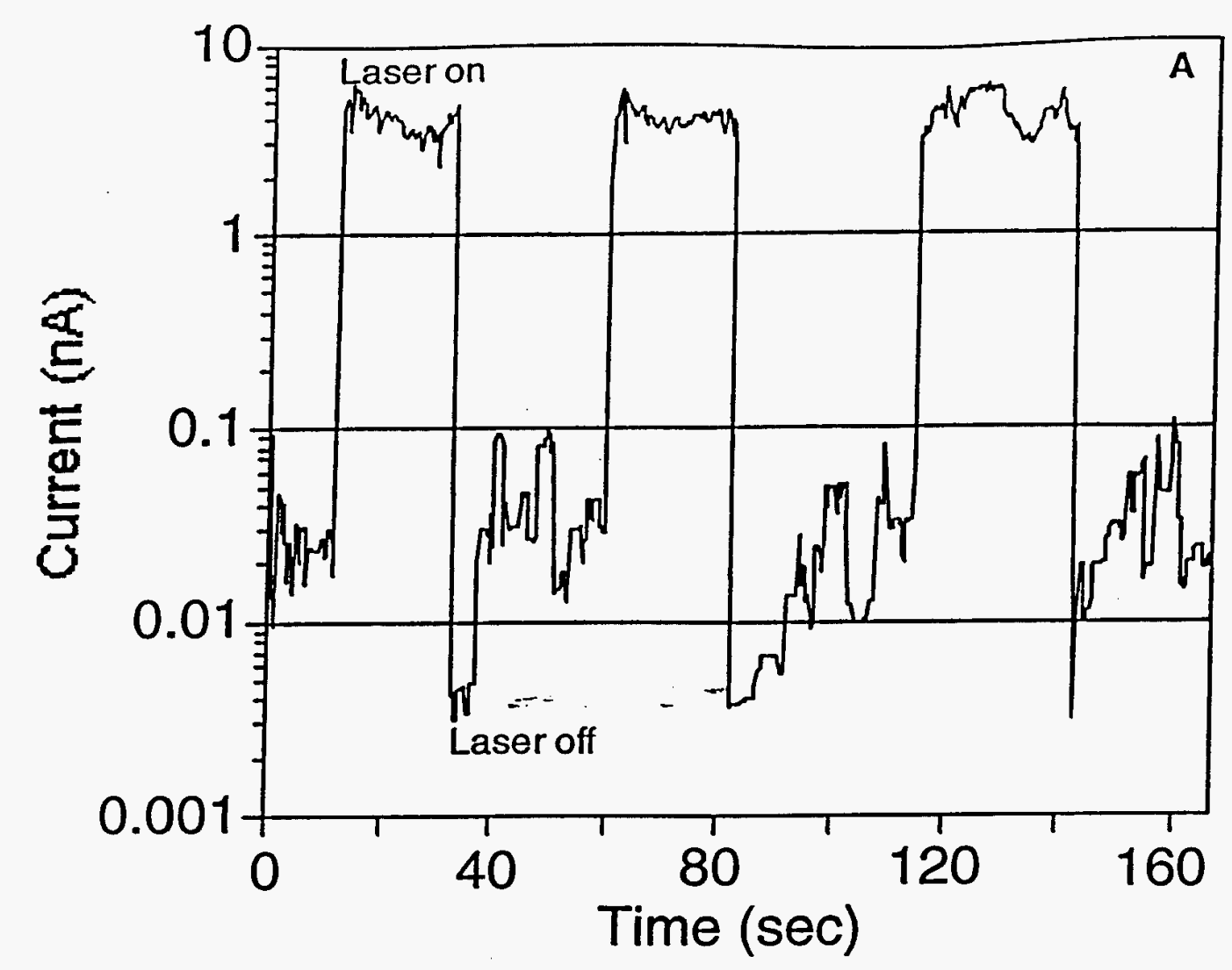

$: z$

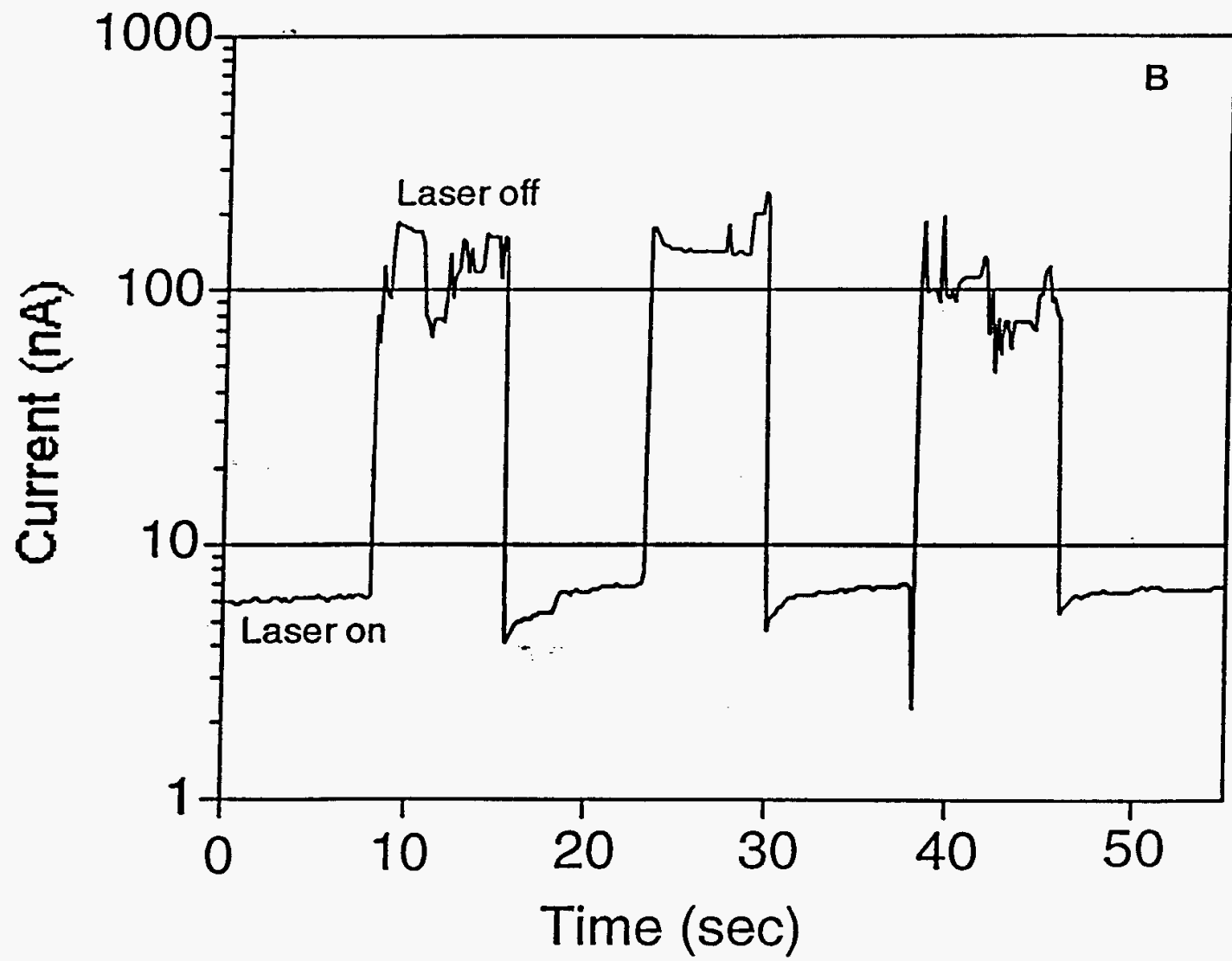

Figure 3. A) Preoxidation and B) Post oxidation two-state behavior in the emission current at a fixed bias voltage of $-80 \mathrm{~V}$ as the sample is laser heated, and when the laser is blocked. The states are indicative of different tip configurations. 
This behavior is made sensible within the following model: after an oxidative etch which opens a closed nanotube, the tip edge atoms rearrange to minimize their energy, tying up dangling bonds in various structures which bridge carbon atoms within and across the edges of the graphene sheets. The marriage of these bridging carbon atoms is frequently tenuous. So much so that under the influence of a strong applied electric field some of these structures break free to project long chains of carbon atoms out from the nanotube tip. Such a chain presents an atomically sharp feature to the field which further concentrates it resulting in a large enhancement in the emission current. This field enhancement is probably often sufficient to rip the chains from the tip, however, the remaining dangling bonds give rise to an energetically unfavorable tip structure. Consequently, rearrangements occur which result in new chain structures forming. A possible mechanism for this chain formation is the unzipping of atoms comprising the edge layers of a helical tube wall. In such a case the maximum number of chains which may be contributed by a single tube wall is precisely equal to its helicity. With laser heating the ability of these chains to remain attached to the tip is diminished so that as soonas such a chain begins to form it is ripped away. Consequently, without the field concentration effected by the long chains the field emission current diminishes to the lower value.

Acknowledgement: This work was supported by the National Science Foundation, the Office of Naval Research, the Robert A. Welch Foundation, and used equipment designed for study of fullerene-encapsulated catalysts supported by the Department of Energy, Division of Chemical Sciences.

\section{REFERENCES}

'R.E. Smalley, Mater. Sci. Eng. B19, 1 (1993).

${ }^{2}$ D.T. Colbert, et al., Science 266, 1218 (1994).

${ }^{3}$ T.W. Ebbesen and P.M. Ajayan, Nature 358, 220 (1992).

'K. Tanaka et al., Chem. Phys. Lett. 223, 65 (1994)

${ }^{5}$ T.W. Ebbesen, P.M. Ajayan, H. Hiura, and K. Tanigaki, Nature 367, 519 (1994).

${ }^{6} \mathrm{O}$. Zhou et al., Science 263, 1744 (1994).

${ }^{7}$ L. Luo, P. Nordlander and R. E. Smalley, submitted to Phys. Rev. Lett. (1994).

${ }^{8}$ T. Guo et al., submitted to Nature (1994).

K.R. Symon, Mechanics, 3rd ed. (Addison-Wesley, Philippines, 1971), pp. 241-247.

${ }_{-}^{10}$ R.H. Fowler and L.W. Nordheim, Roy. Soc. Proc. A119, 173 (1928). 
M9700882

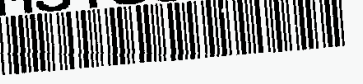

Report Number (14) CONF -9411.44--187

Publ. Date (11) 199411

Sponsor Code (18) $\frac{\text { DUElER }, X F}{U C D-400, D O E / E R}$
UC Category (19)

no 1332.15 form

DOE 